\title{
Mathematical Methods in Quantum Molecular Dynamics
}

\author{
Tucker Carrington (Queen's University) \\ George Hagedorn (Virginia Tech)
}

28 April 2013-3 May 2013

\section{Introduction and Overview of the Field}

The workshop brought together chemists developing mathematical and computational tools for studying the motion of atoms in polyatomic molecules and mathematicians interested in numerical methods for highdimensional problems and semiclassical mechanics. Quantum and semiclassical methods applicable to diatomic molecules are well known and widely used, but the outstanding problem in this field is devising new mathematical and computation tools for studying larger molecules. This is difficult due to the dimensionality of the problem. In principle, molecular dynamics can be understood by solving the time-dependent Schrödinger equation. However, because $3 N$ coordinates are required to specify the configuration of the nuclei in a molecular or reacting system with $N$ atoms, quantum molecular dynamics calculations must deal with very high dimension. This is typically referred to as "the curse of dimensionality." Effective computational approaches exist for solving differential equations in up to three dimensions, but for a molecule with 6 atoms one must deal with 18 dimensions! Three of the 18 coordinates can be chosen to specify the position of the centre of mass of the system, and are therefore easy to separate. It is common to select coordinates so that three others describe the rotational orientation of the system, and if this is done there are $3 N-6$ coordinates describing the shape of the molecule or reacting system. Rotation, however, does not separate because of Coriolis and centrifugal coupling. Although one can easily write down molecular Schrödinger equations, one cannot solve them. So, one resorts to various approximations, primarily to deal with the very high dimensionality of the problem.

At the workshop, mathematicians learned what theoretical chemists are doing and what difficulties they must overcome. Chemists learned about rigorous mathematical results obtained recently by mathematicians. This primarily involved theoretical work, but also included ways to deal with high dimensionality when using computers for approximations.

In prior conferences and workshops in this subject, there have been significant difficulties getting chemists and mathematicians to talk to one another in a meaningful way. There are differences in nomenclature, and people in the two disciplines often have different aims and priorities. A main goal of this workshop was to facilitate as much interaction between the two groups of individuals as possible, and in this regard, the workshop was very successful.

\section{Recent Developments and Open Problems}

The workshop contained talks with emphasis on two different areas. One involved how to deal directly with the difficulties of high dimensional problems. The other dealt with theoretical results in the mathematics of Schrödinger operators. 


\subsection{Mathematical Methods for High-Dimensional Problems}

To solve the Schrödinger equation numerically in order to compute a vibrational or ro-vibrational spectrum, one often expands the wavefunctions of interest as linear combinations of known basis functions [1, 2, 3, 4]. The expansion coefficients are typically obtained as components of eigenvectors of the basis representation of the Hamiltonian operator. To make it possible to deal with large basis sets, it is now common to use iterative numerical methods (e.g., Lanczos, Davidson, Filter Diagonalization) to compute eigenvalues and eigenvectors of the Hamiltonian matrix by evaluating matrix-vector products $[3,4,5,6,7,8,9,10,11,12$, $13,14,15]$. With a (direct or nondirect) product basis, these ideas can be applied efficiently, regardless of the form and complexity of the potential energy surface (PES), provided that a direct product quadrature is used. $[16,14,17,18,19,20,21,22,23,24,25]$.

Iterative methods require storing vectors, but not the Hamiltonian matrix. However, for molecules with six or more atoms, the size of the vectors one must manipulate becomes large (compared to the memory of typically available computers). For molecules with five atoms, calculations of this kind are now possible [26]. One way to deal with the basis-size problem is to use basis functions that are not products of functions of a single variable $[27,28,29,30,23,24,20,31,32,33,21]$. Another strategy is to use product functions but only a fraction of the full product basis $[34,35,36,37,38,39,40,41,42]$. Often many of the functions in a product basis have large zeroth order energies. They are therefore superfluous and can be discarded. Once a product basis has been pruned, one needs new ideas for evaluating the matrix-vector products required to obtain the spectrum. This is due to the fact that well-established techniques for evaluating matrix vector products exploit the structure of both the basis and the quadrature grid [3, 4].

A popular way to deal with the basis size explosion problem is to use optimized basis functions to build the product functions. This is implemented in the multi configuration time dependent hartree approach (MCTDH). Manthe, Meyer, Lubich, and Wang all presented talks about MCTDH at the workshop. MCTDH is only practical with more than about 6 coordinates if one uses "logical" coordinates that lump together groups of the actual coordinates. This is often implemented with a tree structure, as discussed by Manthe and Schneider at the workshop.

If one uses a pruned basis, there are three options for evaluating potential matrix-vector products. The first obviates the need for multi-dimensional quadrature by writing the potential as a sum of terms, each of which is a product of functions of a single variable [43, 44]. At the workshop, Manzhos presented a new method for doing this. Matrix-vector products are evaluated term by term. If the basis pruning is done so that some basis set structure is retained, the matrix-vector products can be efficiently evaluated regardless of the sparsity of the matrix representations of the terms [40]. Good schemes for fitting potentials as sum of products do exist [45, 46]. Most (but not all [47]) multiconfiguration time dependent Hartree calculations require a sum-of-products potential [43]. A problem with this option is that the cost scales linearly with the number of terms in the potential. Tensor decomposition methods presented by Schneider and Martinez and Meyer can be used to make potentials with sum of products form.

A second option is to re-represent the potential as a sum of terms depending on one, two, three, etc. coordinates (called a multimode expansion by chemists and an ANOVA expansion by mathematicians). One then exploits the fact that, with product basis functions, many of the matrix elements of these terms are zero $[34,35,48,49,50]$. At the workshop Bowman and Griebel discussed implementations of this strategy. For many molecules, and even for some unimolecular and bimolecular reactions, approximating the potential as a sum of terms depending on four or five coordinates is accurate enough that good results can be obtained [51]. Many of the matrix elements of the lower-dimensional functions are zero, and this facilitates computing and storing their matrix representations (either on disk [35] or in memory [49]). It also means that the Hamiltonian matrix is sparse, and this sparsity could be exploited when evaluating matrix-vector products. This is an excellent approach. However, as the number of terms in the potential (the order of the mode representation) increases, the number of matrix elements to compute and store also increases, and the sparsity of the Hamiltonian matrix decreases. This makes it costly to check the accuracy of the approximation to the potential. It is, nonetheless, true that (if the basis is large enough) accurate results can be obtained by retaining enough terms in the multimode expansion. If $3 N-6$ dimensional terms are retained ( $N$ is the number of atoms) the multimode representation of the potential is exact.

A third option is to use full-dimensional quadrature. Related ideas were presented at the workshop by Avila, Carrington, and Lauvergnat. When quadrature is used, all potential terms are treated together, and there 
is no need to evaluate matrix-vector products term by term. There is also no need to approximate the potential as a sum of terms depending on one, two, three, etc coordinates [52,53,54]. When one uses the full potential, and therefore multidimensional quadrature, the size of the quadrature grid is an important problem. By using ideas based on those of Smolyak [55, 56, 57, 58, 59, 60, 61, 62, 63, 64, 65, 66, 67, 68, 69, 70, 71, 72, 73], it is, nevertheless, possible to compute accurate energy levels using grids that are many orders of magnitude smaller than direct product grids.

\subsection{Mathematical Results for Schrödinger Operators}

Almost all results in this subject make use of the Born-Oppenheimer approximation. This allows one to separate nuclear and electronic motions, but the approximation relies on assumptions that may be violated in some molecules. In particular, traditional Born-Oppenheimer approximations fail at level crossings and avoided crossings (configurations of the nuclei at which two electronic energy levels are equal or very close to one another).

There are now several approaches for dealing with level crossings and avoided crossings, for example $[99,100,101,102,103,104]$. At this workshop, talks by Betz and Goddard presented a recent approach for dealing with these problems. The talk by Fermanian was also dealt with these problems.

Interesting topological quantities arise from level crossings. The talk by Panati showed that very sophisticated ideas from differential geometry and topology may be required to describe the structures that can arise.

In Born-Oppenheimer approximations, the nuclear motion is typically described by semiclassical mechanics. The talk by Joye described exponentially accurate semiclassical techniques that have recently been developed to describe tunneling and above barrier reflections. These phenomena are quantum mechanical and are not present in classical mechanics. Other talks involving semiclassical approximations were presented by Jennings, Jin, Jefferis, and Miller.

The workshop also featured talks that involved mathematical modeling of certain molecular phenomena. For example, the talk by Hagedorn described a simple model for molecular Rayleigh and Raman scattering stimulated by a short laser pulse. The talk by Tremblay described interaction of a $C O$ molecule with various metal surfaces. The talk by Teufel described a mathematical model for a molecule interacting with a quantized radiation field.

Talks by Christiansen and Martinez described tensor decompositions that have recently been introduced in numerical work in an attempt to deal with the high dimensionality of molecular dynamics calculations.

\section{Scientific Progress Made}

As indicated in the introduction, a major goal of this workshop was the sharing of information between mathematicians and chemists. Many participants commented that this meeting was much more successful than earlier such meetings that brought the two groups of researchers together. The two groups often have closely related goals, but they publish their results in different journals and often one group has no idea of progress made by the other group.

The speakers from each group made an effort to address the other group, which often does not happen in such meetings. Also, some speakers commented that they welcomed the opportunity to present their work to audiences they had never addressed before.

Both groups gained insight into activities and scientific difficulties encountered by the other group.

\section{Outcome of the Meeting}

Ideally, this workshop would lead to new projects for participants and perhaps some new collaborations. It is too early to know the extent to which those things are happening. However, the organizers have heard of several interactions that resulted from the meeting. For example, Hagedorn, Valeev, and Miller have been communicating about Raman scattering, Also, Lasser and Fermanian have begun working on a project that was inspired by the talk of Burghardt. 
We expect that the sharing of information at the workshop will lead to participants developing future projects. Often the most important part of research is choosing good problems on which to work. With the sharing of information between the mathematicians and chemists, members of both groups will be aware of new opportunities.

Several participants asked the organizers to submit a new proposal to try to hold a similar meeting in 2015. Also, George Hagedorn and Caroline Lasser are contemplating submitting a proposal to the Mathematisches Forschungsinstitut Oberwolfach to try to hold a meeting, with the sharing of information between mathematicians and chemists as a main goal.

\section{Fundamental open questions}

Mathematical analysis is often based on either the assumption that $\hbar$ is small or that some ratio of masses is small. In the former case semiclassical approximations work well, and in the latter cases adiabatic approximations are appropriate. Mathematicians are working on ways to improve and correct both of these and to deal with level crossings where adiabatic approximations fail. It is important to know whether the corrections enable one to achieve the sort of accuracy desired by chemists. In the application of Smolyak methods to problems of chemical interest, more work is needed to develop ideas to exploit symmetry. It is also important to develop good black-box methods that can be used by non-experts.

\section{References}

[1] S. Carter and N. C. Handy, Comp. Phys. Rep. 5, 115 (1986).

[2] J. Tennyson, Comp. Phys. Rep. 4, 1 (1986).

[3] J.C. Light and T. Carrington Jr., Adv. Chem. Phys. 114, 263 (2000).

[4] J. Bowman, T. Carrington, H.-D. Meyer, Mol. Phys. 106, 2145 (2008).

[5] A. McNichols and T. Carrington, Jr., Chem. Phys. Lett. 202, 464 (1993).

[6] M.J. Bramley and T. Carrington Jr, J. Chem. Phys. 99, 8519 (1993).

[7] D. Neuhauser, J. Chem. Phys. 93, 2611 (1990)

[8] R.B. Lehoucq, S.K. Gray, D.-H. Zhang, and J.C. Light, Comput. Phys. Commun. 109, 15 (1998).

[9] F. Ribeiro, C. Iung, and C. Leforestier, J. Chem. Phys. 123, 054106 (2005)

[10] C. Iung and C. Leforestier, J. Chem. Phys. 90, 3198 (1989) .

[11] S. Huang and T. Carrington Jr., Chem. Phys. Lett. 312 311-318 (1999).

[12] G. Balint-Kurti and P. Pulay J. Molec. Struc. (Theochem) 341, 1 (1995).

[13] D. Xu, , R. Chen, and H. Guo, J. Chem. Phys. 118, 7273 (2003)

[14] R. Chen, G. Ma and H. Guo, J. Chem. Phys. 114, 4763 (2001)

[15] H.G. Yu, Chem. Phys. Lett. 281, 312 (1997)

[16] M. J. Bramley, J. W. Tromp, T. Carrington Jr., and G. C. Corey, J. Chem. Phys. 100, 6175 (1994).

[17] L. Zheng, Y. Lu, S.-Y. Lee, H. Fu, and M. Yang, J. Chem. Phys. 134, 054311 (2011)

[18] X.-G. Wang and T. Carrington, Jr, J. Chem. Phys. 115, 9781 (2001).

[19] X.-G. Wang and T. Carrington, Jr, J. Chem. Phys. 118, 6946 (2002). 
[20] X.-G. Wang and T. Carrington, Jr., J. Chem. Phys. 121, 2937 (2004)

[21] X.-G. Wang and T. Carrington, Jr., J. Chem. Phys. 129, 234102 (2008).

[22] X.-G. Wang, T. Carrington, J. Tang, A.R.W. McKellar, J. Chem. Phys. 123034301 (2005)

[23] X.-G. Wang and T. Carrington, Jr, J. Chem. Phys. 119, 101 (2003).

[24] J.C. Tremblay and T. Carrington, Jr., J. Chem. Phys. 125, 094311 (2006)

[25] P. Sarkar, N. Poulin, T. Carrington Jr., J. Chem. Phys. 11010269 (1999)

[26] E. Mtyus, G. Czakó, B.T. Sutcliffe, and A.G. Császár, J. Chem. Phys. 127084102 (2007).

[27] M. J. Bramley and T. Carrington, Jr., J. Chem. Phys. 101, 8494 (1994).

[28] A. Viel and C. Leforestier, J. Chem. Phys. 112, 1212 (2000).

[29] R. A. Friesner, J.A. Bentley, M. Menou, and C. Leforestier, J. Chem. Phys. 99, 324 (1993).

[30] X.-G. Wang and T. Carrington, Jr, J. Chem. Phys. 117, 6923 (2002).

[31] H.-G. Yu, J. Chem. Phys. 117, 8190 (2002).

[32] H.-S. Lee and J.C. Light, J. Chem. Phys. 118, 3458 (2003)

[33] H.-S. Lee and J.C. Light, J. Chem. Phys. 120, 4626 (2004)

[34] S. Carter, S.J. Culik, J.M. Bowman, J. Chem. Phys. 107, 10458 (1997).

[35] J. M. Bowman, S. Carter, and X. Huang, Int. Rev. Phys. Chem. 22, 533 (2003).

[36] D.T. Colbert and W.H. Miller J. Chem. Phys. 96, 1982 (1992)

[37] H.-G. Yu, J. Chem. Phys. 117, 2030 (2002)

[38] B. Poirier, J. Theor. Comput. Chem. 2, 65 (2003).

[39] D. Bégué, N. Gohaud, C. Pouchan, P. Cassam-Chenai, and J. Liévin, J. Chem. Phys. 127, 164115 (2007)

[40] X.-G. Wang and T. Carrington, Jr., J. Phys. Chem. A, 105, 2575 (2001)

[41] J. Cooper and T. Carrington, Jr., J. Chem. Phys. 130214110 (2009).

[42] R. Dawes and T. Carrington Jr., J. Chem. Phys. 122134101 (2005).

[43] M. H. Beck, A. Jäckle, G. A. Worth, and H.-D. Meyer, Phys, Rep. 3241 (2000).

[44] H. Romanowski, J. Bowman, and L. Harding, J. Chem. Phys. 82, 4155 (1985).

[45] A. Jaeckle and H.-D. Meyer. J. Chem. Phys. 104, 7974 (1996).

[46] S. Manzhos and T. Carrington, Jr., J. Chem. Phys. 125194105 (2006).

[47] U. Manthe, J. Chem. Phys. 105, 6989 (1996).

[48] S. Carter, J. M. Bowman, and N. C. Handy, Theor. Chim. Acta 100, 191 (1998).

[49] D.M. Benoit, J. Chem. Phys. 120, 562 (2004).

[50] P. Meier, M. Neff, and G. Rauhut, J. Chem. Theory and Comp. 7, 148 (2011).

[51] J. Wu, X. Huang, S. Carter, J.M. Bowman, Chem. Phys. Lett. 426285 (2006).

[52] G. Avila and T. Carrington, Jr., J. Chem. Phys. 131, 174103 (2009). 
[53] G. Avila and T. Carrington, Jr., J. Chem. Phys. 134, 054126 (2011).

[54] G. Avila and T. Carrington, Jr., J. Chem. Phys. 135, 135, 064101 (2011).

[55] S. A. Smolyak, Sov. Math. Dokl. 4, 240 (1963).

[56] K. Frank, S. Heinrich, and S. Pereverzev, J. Complex. 12, 17(1996).

[57] A.C. Genz, SIAM Soc. Ind. Appl. Math. J. Numer. Anal. 23, 1273(1986).

[58] G.W. Wasilkowski and H. Woniakowski, J. Complex. 11, 1 (1995).

[59] H. Yserentant, Numerische Mathematik, 49 379-412,(1986).

[60] F.-J. Delvos and W. Schempp, Pitman Res. Notes Math., 230 , Longman (1989).

[61] M. Griebel, P. Oswald, and T. Schiekofer, Numer. Mathematik, 83 (2):279312, 1999

[62] M. Griebel, Computing, 61 (2):151179, 1998.

[63] C. Schwab and R. A. Todor, Numer. Math., 95 (4):707734, 2003.

[64] M. Griebel, M. Schneider, Ch. Zenger, "A combination technique for the solution of sparse grid problems" R. Beauwens (ed.) P. de Groen (ed.), Iterative Methods in Linear Algebra , Elsevier \& NorthHolland, 263 (1992)

[65] J. Garcke and M. Griebel, Journal of Computational Physics, 165 (2):694716, 2000.

[66] V.N. Temlyakov, "Approximation of periodic functions", Nova Science (1994).

[67] M. Griebel and J. Hamaekers, Tensor product multiscale many-particle spaces with finite-order weights for the electronic Schrdinger equation. In M. Dolg, editor, Modern and Universal First-principles Methods for Many-electron Systems in Chemistry and Physics, volume 3 of Progress in Physical Chemistry, pages 237-253. Oldenbourg Wissenschaftsverlag GmbH, Mnchen, 2010.

[68] V. Barthelmann, E. Novak, and K. Ritter, Adv. Comput. Math. 12 (4): 273-288 (2000)

[69] E. Novak, and K. Ritter, R. Schmitt, and A. Steinbauer, Journal of Computational and Applied Mathematics, 112 n.1-2, 215-228, Nov. 30, 1999

[70] E. Novak and K. Ritter, Numer. Math. 75, 79 (1996).

[71] D. Lauvergnat and A. Nauts, Phys. Chem. Chem. Phys., 128405 (2010)

[72] J.I. Rodriguez, D.C. Thompson, J.S.M Anderson, J.W. Thomson and P.W. Ayers, J. Phys. A: Math. Theor. 41365202 (2008).

[73] J.I. Rodriguez, D.C. Thompson, P. W. Ayers, and A. Koester, Journal of Chemical Physics, 128224103 (2008).

[74] M.-L. Senent, P. Palmieri, S. Carter and N.C. Handy, Chem. Phys. Lett. 354, 1 (2002).

[75] D.P. Tew, N.C. Handy and S. Carter, Mol. Phys. 99, 393 (2001).

[76] Y. Wang, B.J. Braams, J.M. Bowman, S. Carter and D. Tew, J. Chem. Phys. 128, 224314 (2008).

[77] A.L. Kaledin and J.M. Bowman, J. Phys. Chem. A 111, 5593 (2007).

[78] S. Carter, J.M. Bowman, and N.C. Handy Molec. Phys. 110, 775 (2012).

[79] S. Carter, J.M. Bowman, L.B. Harding, Spectrochimica Acta A 531179 (1997).

[80] S. Carter, N.C. Handy, Chem. Phys. Lett. 3521 (2002) 
[81] J.O. Jung and R.B. Gerber, J. Chem. Phys. 105, 10332 (1996).

[82] H. Rabitz, O. F. Alis, J. Shorter, and K. Shim, Comput. Phys. Commun. 117, 11 (1999).

[83] G. Rauhut, J. Chem. Phys. 1219313 (2004)

[84] J.N. Murrell, S. Carter, S. Frantos, P. Huxley, A.J.C. Varandas, Molecular Potential Energy Functions, John Wiley \& Sons, Toronto, 1984.

[85] I.M. Sobol, Reliability Eng. Syst. Safety 79187 (2003)

[86] Christian Lubich, From Quantum to Classical Molecular Dynamics: Reduced Models and Numerical Analysis (Zurich Lectures in Advanced Mathematics) (2008)

[87] Stuart Carter, private communication.

[88] S. Carter and J.M. Bowman, J. Chem. Phys., preprint.

[89] J. M. L. Martin, T. J. Lee, P. R. Taylor, and J. P. Francois, J. Chem. Phys. 103, 2589 (1995).

[90] M. Griebel and S. Knapek, Mathematics of Computations, 78, 2223 (2009); Constructive Approximation, 16 525, (2000)

[91] J. S. M. Anderson and P. W. Ayers, 2nd Workshop on Sparse Grids and Applications, Munich, 2012.

[92] H.-J. Bungartz and M. Griebel, Acta Numerica 13, 147 (2004).

[93] F. Heiss, Viktor Winschel, Journal of Econometrics, 144, 62 (2008).

[94] “Quantities, Units and Symbols in Physical Chemistry," IUPAC Green Book, 3rd Edition IUPAC \& RSC Publishing, Cambridge (2008)

[95] M. Mladenovic, J. Chem. Phys. 137, 014306 (2012)

[96] See Supplementary Material Document No. for energy levels of all symmetries.

[97] O. Christiansen, Phys. Chem. Chem. Phys. 9, 2942 (2007).

[98] O. Christiansen and J. Luis Int. J. Quant. Chem. 104, 667 (2005).

[99] G.A. Hagedorn, Memoirs Amer. Math. Soc. 111 (536), 1-130 (1994).

[100] G.A. Hagedorn and A. Joye, Ann. Inst. H. Poincaré 68, 85-134 (1998).

[101] G.A. Hagedorn and A. Joye, Rev. Math. Phys. 11, 41-101 (1999).

[102] S. Teufel, Adiabatic Perturbation Theory in Quantum Dynamics. Lecture Notes in Mathematics 1821. Springer-Verlag, Berlin, Heidelberg, New York, 2003.

[103] C. Fermanian-Kammerer, Reviews in Mathematical Physics, 15, 1285-1317 (2003).

[104] C. Lasser and S. Teufel, Comm. Pure Appl. Math. 58, 1188-1230 (2005) 